\title{
Simultaneous exact control of piezoelectric systems in multilayered media
}

\author{
BORIS V. KAPITONOV ${ }^{1 *}$ and MARCO ANTONIO RAUPP ${ }^{2}$ \\ ${ }^{1}$ Sobolev Institute of Mathematics, Siberian Branch of Russian Academy of Sciences \\ ${ }^{2}$ Laboratory of Scientific Computation, LNCC/MC \\ 25651-070 Quitandinha, Petrópolis, RJ, Brasil \\ E-mail: borisvk@lncc.br
}

\begin{abstract}
This paper considers a pair of transmission problems for the system of piezoelectricity having piecewise constant coefficients. Under suitable monotonicity conditions on the coefficients and certain geometric conditions on the domain and the interfaces where the coefficients have a jump discontinuity, results on simultaneous boundary observation and simultaneous exact control are established.
\end{abstract}

Mathematical subject classification: 35L50; 35Q60; 35B40.

Key words: Piezoelectricity; transmission problem; simultaneous exact controllability.

\section{Introduction}

Throughout this paper $\Omega$ will be a bounded domain in $\mathbb{R}^{3}$ with sufficiently smooth boundary $S$. For $k=1,2, \cdots, n$, let $\mathcal{B}_{k}$ be open, bounded and connected subsets of $\Omega$ with smooth boundary $S_{k}$, and such that $\overline{\mathcal{B}}_{k} \subset \mathcal{B}_{k+1}$. We set

$$
\Omega_{0}=\mathcal{B}_{1}, \quad \Omega_{k}=\mathcal{B}_{k+1} \backslash \overline{\mathcal{B}}_{k} \text { for } k=1,2, \cdots, n-1, \quad \Omega_{n}=\Omega \backslash \overline{\mathcal{B}}_{n} .
$$

Assume that $\Omega$ is occupied by a linear multilayered piezoelectric body whose motion is governed by the following system ([4], [6])

$$
\begin{cases}\rho \ddot{u}=\nabla \cdot T, & \mu \dot{H}=-\operatorname{curl} E, \quad \dot{\mathcal{D}}=\operatorname{curl} H, \\ \nabla \cdot \mathcal{D}=0, & \nabla \cdot H=0, \\ T=c \cdot \widehat{\nabla} u-E \cdot e, & \mathcal{D}=e \cdot \widehat{\nabla} u+b \cdot E,\end{cases}
$$

\#555/02. Received: 17/IX/02.

*Supported by FAPERJ (Brazil), project E-26/151.523/01. Visiting Researcher at the National Laboratory of Scientific Computation (LNCC/MCT). 
where $\rho$ is the mass density, $u$ is the displacement vector, $\widehat{\nabla} u$ is the symmetric part of $\nabla u, T$ is the stress tensor, $H$ is the magnetic field, $E$ is the electric field, $\mathcal{D}$ is the electric displacement vector, $\mu$ is the magnetic permeability, $c, e, b$ are the elastic, piezoelectric and electric permittivity tensors respectively whose Cartesian components satisfy the following properties:

$$
\begin{array}{ll}
c_{i j k l}=c_{j i k l}=c_{k l i j}, & b_{i j}=b_{j i}, \\
b_{i j} \xi^{j} \xi^{i} \geq b_{0}|\xi|^{2}, & b_{0}>0
\end{array}
$$

for any real vector $\xi=\left(\xi^{1}, \xi^{2}, \xi^{3}\right) \in \mathbb{R}^{3}$,

$$
c_{i j k l} \varepsilon_{k l} \varepsilon_{i j} \geq c_{0} \varepsilon_{i j} \varepsilon_{i j}, \quad c_{0}>0
$$

for any real symmetric matrices $\left\{\varepsilon_{i j}\right\}$ of order 3 .

We introduce the following matrices of order 3

$$
A_{i}=\left\{e_{k h i}\right\}, \quad B=\left\{b_{k h}\right\}, \quad A_{i j}=\left\{a_{k h}^{i j}\right\},
$$

where

$$
a_{k h}^{i j}=\left(1-\delta_{i h} \delta_{j k}\right) c_{i k j h}+\delta_{i k} \delta_{j h} c_{i h j k} .
$$

It follows from the symmetry of $c_{i j k l}$ that

$$
A_{i j}^{*}=A_{j i}
$$

Using these notations, we write the system (1.1) in the matrix form

$$
\left\{\begin{array}{l}
\rho \frac{\partial^{2} u}{\partial t^{2}}-\frac{\partial}{\partial x_{i}}\left\{A_{i j} \frac{\partial u}{\partial x_{j}}-A_{i}^{*} E\right\}=0, \\
\frac{\partial}{\partial t}\left\{B E+A_{i} \frac{\partial u}{\partial x_{i}}\right\}-\operatorname{curl} H=0, \\
\mu \frac{\partial H}{\partial t}+\operatorname{curl} E=0, \\
\operatorname{div}\left\{B E+A_{i} \frac{\partial u}{\partial x_{i}}\right\}=0, \quad \operatorname{div} H=0 .
\end{array}\right.
$$


We note that

$$
\frac{\partial}{\partial x_{i}}\left\{A_{i j} \frac{\partial u}{\partial x_{j}}\right\}=\frac{\partial}{\partial x_{j}}\left\{c_{1 j k h} \varepsilon_{k h}(u), c_{2 j k h} \varepsilon_{k h}(u), c_{3 j k h} \varepsilon_{k h}(u)\right\},
$$

where

$$
\varepsilon_{k h}(u)=\frac{1}{2}\left(\frac{\partial u^{k}}{\partial x_{h}}+\frac{\partial u^{h}}{\partial x_{k}}\right)
$$

It is assumed that

$$
\sum_{i, j=1}^{3}\left(A_{i j} \eta_{j}, \eta_{i}\right) \geq C_{0} \sum_{i=1}^{3}\left|\eta_{i}\right|^{2}, \quad C_{0}>0
$$

for any real vector $\eta_{i} \in \mathbb{R}^{3}$. Here $(\cdot, \cdot)$ denotes the inner product in $\mathbb{R}^{3}$. We remark that this assumption holds for an isotropic medium $\left(c_{i j k h}=\tilde{\lambda} \delta_{i j} \delta_{k h}+\right.$ $\tilde{\mu} \delta_{i k} \delta_{j h}+\tilde{\mu} \delta_{i h} \delta_{j k}$ ) with a constant $C_{0}=\tilde{\mu}$ :

$$
\sum_{i, j=1}^{3}\left(A_{i j} \eta_{j}, \eta_{i}\right)=(\tilde{\lambda}+\tilde{\mu})\left(\sum_{i=1}^{3} \eta_{i}^{i}\right)^{2}+\tilde{\mu} \sum_{i, j=1}^{3}\left(\eta_{i}^{j}\right)^{2} \geq \tilde{\mu} \sum_{i=1}^{3}\left|\eta_{i}\right|^{2} .
$$

It is assumed that $c_{i j k h}(x), b_{i j}(x), \mu(x)$ are piecewise constant functions which lose the continuity on $S_{1}, S_{2}, \cdots, S_{n}, \rho$ and $e_{k h i}$ are constants, $\rho>0$.

We consider the following transmission problems

$$
\left\{\begin{array}{l}
\rho \frac{\partial^{2} u}{\partial t^{2}}-\frac{\partial}{\partial x_{i}}\left\{A_{i j} \frac{\partial u}{\partial x_{j}}-A_{i}^{*} E\right\}=0, \\
\frac{\partial}{\partial t}\left\{B E+A_{i} \frac{\partial u}{\partial x_{i}}\right\}-\operatorname{curl} H=0, \\
\quad(x, t) \in \Omega_{m} \times(0, T) m=0,1, \cdots, n \\
\mu \frac{\partial H}{\partial t}+\operatorname{curl} E=0, \\
\operatorname{div}\left\{B E+A_{i} \frac{\partial u}{\partial x_{i}}\right\}=0, \\
\quad \operatorname{div} H=0 .
\end{array}\right.
$$




$$
\left\{\begin{array}{c}
u^{(m-1)}=u^{(m)},\left(A_{i j}^{(m-1)} \frac{\partial u^{(m-1)}}{\partial x_{j}}-A_{i}^{*} E^{(m-1)}\right) v_{i} \\
=\left(A_{i j}^{(m)} \frac{\partial u^{(m)}}{\partial x_{j}}-A_{i}^{*} E^{(m)}\right) v_{i}, \\
(x, t) \in S_{m} \times(0, T), \quad m=1, \cdots, n \\
{\left[v, E^{(m-1)}\right]=\left[\nu, E^{(m)}\right],\left[v, H^{(m-1)}\right]=\left[v, H^{(m)}\right],} \\
\left\{\left(A_{i j} \frac{\partial u}{\partial x_{j}}-A_{i}^{*} E\right) v_{i}+\beta u\right\} \mid s \times(0, T)=Q(x, t), \\
{\left.[E, v]\right|_{S \times(0, T)}=G(x, t) .}
\end{array}\right.
$$

and

$$
\begin{aligned}
& \rho \frac{\partial^{2} v}{\partial t^{2}}-\frac{\partial}{\partial x_{i}}\left\{A_{i j} \frac{\partial v}{\partial x_{j}}-A_{i}^{*} \Phi\right\}=0 \\
& \frac{\partial}{\partial t}\left\{B \Phi+A_{i} \frac{\partial v}{\partial x_{i}}\right\}-\operatorname{curl} \Psi=0 \\
& (x, t) \in \Omega_{m} \times(0, T) \quad m=0,1, \cdots, n \\
& \mu \frac{\partial \Psi}{\partial t}+\operatorname{curl} \Phi=0 \\
& \operatorname{div}\left\{B \Phi+A_{i} \frac{\partial v}{\partial x_{i}}\right\}=0, \quad \operatorname{div} \Psi=0 \\
& \left.v\right|_{t=0}=\varphi_{1}(x),\left.\quad \frac{\partial v}{\partial t}\right|_{t=0}=\varphi_{2}(x),\left.\quad \Phi\right|_{t=0}=\varphi_{3}(x),\left.\quad \Psi\right|_{t=0}=\varphi_{4}(x), \\
& \left\{\begin{array}{c}
v^{(m-1)}=v^{(m)},\left(A_{i j}^{(m-1)} \frac{\partial v^{(m-1)}}{\partial x_{j}}-A_{i}^{*} \Phi^{(m-1)}\right) v_{i} \\
=\left(A_{i j}^{(m)} \frac{\partial v^{(m)}}{\partial x_{j}}-A_{i}^{*} \Phi^{(m)}\right) v_{i}, \\
(x, t) \in S_{m} \times(0, T), \quad m=1, \cdots, n \\
{\left[\nu, \Phi^{(m-1)}\right]=\left[\nu, \Phi^{(m)}\right],\left[\nu, \Psi^{(m-1)}\right]=\left[\nu, \Psi^{(m)}\right],}
\end{array}\right.
\end{aligned}
$$




$$
\left.v\right|_{s \times(0, t)}=\mathcal{D}(x, t),\left.\quad[\Psi, v]\right|_{s \times(0, T)}=\mathcal{P}(x, t),
$$

where $[\cdot, \cdot]$ is the vector product, $v=v(x)=\left(v_{1}, v_{2}, v_{3}\right)\left(\right.$ for $x \in S_{m}, x \in S$ ) is the unit normal vector pointing into the exterior of $\mathcal{B}_{m}$ or $\Omega ; A_{i j}^{(m)}, u^{(m)}, v^{(m)}$, $E^{(m)}, \Phi^{(m)}, H^{(m)}, \Psi^{(m)}$ are the restrictions of the corresponding matrices and vector-functions on $\Omega_{m}$. In (1.5) $\beta=\beta(x)$ is a continuously differentiable positive function on $S$.

The problem of exact boundary control for the system (1.2)-(1.5) ((1.6)-(1.9)) is formulated as follows:

Given the initial distribution $f=\left\{f_{1}, f_{2}, f_{3}, f_{4}\right\}\left(\varphi=\left\{\varphi_{1}, \varphi_{2}, \varphi_{3}, \varphi_{4}\right\}\right)$, time $T>0$, and a desired terminal state $g=\left\{g_{1}, g_{2}, g_{3}, g_{4}\right\}\left(\psi=\left\{\psi_{1}, \psi_{2}, \psi_{3}, \psi_{4}\right\}\right)$ with $f, g(\varphi, \psi)$ in appropriate function spaces, find a vector-valued functions $Q(x, t), G(x, t)(\mathcal{D}(x, t), \mathcal{P}(x, t))$ in a suitable function spaces such that the solution of (1.2)-(1.5) ((1.6)-(1.9)) satisfies the conditions

$$
\left.\left\{u, \frac{\partial u}{\partial t}, E, H\right\}\right|_{t=T}=g(x)\left(\left.\left\{v, \frac{\partial v}{\partial t}, \Phi, \Psi\right\}\right|_{t=T}=\psi(x)\right) .
$$

Our purpose is to obtain simultaneous exact boundary control of these problems, $\{\mathcal{D}(x, t), \mathcal{P}(x, t)\}$ serving as a control in problem (1.6)-(1.9), while the vector-valued functions

$$
Q(x, t)=\frac{\partial}{\partial t} \mathcal{D}(x, t), G(x, t)=[v, \mathcal{P}(x, t)]
$$

is a control in (1.2)-(1.5).

Spatial energy estimates for a semi-infinite piezoelectric beam have been studied by A. Borrelli and M.C. Patria [2].

Boundary controllability for some partial case of the system (1.1) with another boundary and interface conditions was investigated in [14].

For $A_{i} \equiv 0$, the piezoelectric system (1.2) decouples into a pair of hyperbolic systems: the Maxwell system and the hyperbolic system of second order.

The exact controllability problem for the Maxwell system has been studied by D. Russell [27] for a circular cylindrical region, by K. Kime [16] for a spherical region, and by J. Lagnese [20] for a general region. Stabilization for the Maxwell system with the Silver-Müller absorbing boundary conditions and exact controllability for corresponding initial boundary value problem have been studied by 
V. Komornik [17], P. Martinez [24] and N. Weck [28]. The uniform exponential decay of solutions of Maxwell's equations with boundary dissipation and exact boundary controllability was proved in [7], [8].

Stabilization and exact boundary controllability for the system of elasticity have been studied by J. Lagnese [18], [19], F. Alabau and V. Komornik [1] and M. Horn [5] among others. In [7], [9] boundary observation, stabilization and exact controllability were studied for a class of hyperbolic systems which includes the system of elasticity.

Boundary controllability in transmission problems for a class of second order hyperbolic systems has been studied by J. Lagnese [19]. Uniform stabilization and exact control for the Maxwell system in multilayered media were investigated in [8]. The question of boundary controllability in transmission problems for the wave equation has been considered by J.-L. Lions [23], and S. Nicaise [25], [26].

The main novelty of this note is that we study the simultaneous exact control. Simultaneous exact control for the wave equation has been established by D. Russell [27] for a circular cylindrical region and by J.-L. Lions [22], F. Khodja and A. Bader [15].

In [9]-[13] simultaneous controllability were studied for a class of hyperbolic systems of second order, for a pair of Maxwell's equations and for a class of evolution systems which includes the Schrödinger equation.

This article is organized as follows: simultaneous boundary observation for the problems (1.2)-(1.5) and (1.6)-(1.9) with zero boundary conditions $(Q \equiv G \equiv$ $\mathcal{D} \equiv \mathcal{P} \equiv 0$ ) is established in Section 2. In Section 3 the simultaneous exact controllability is studied by means of the Hilbert Uniqueness Method, introduced by J.-L. Lions [21], [22].

\section{Boundary observability}

Throughout this paper $H_{k}(\Omega)$ and $H_{q}(S)$ denote the usual Sobolev spaces.

We denote by $\mathcal{H}_{0}$ the Hilbert space of pairs $u=\left\{u_{1}(x), u_{2}(x)\right\}$ of threecomponent vector-valued functions

$$
u_{i} \in L_{2}(\Omega), \operatorname{curl} u_{i} \in L_{2}\left(\Omega_{m}\right), m=0,1, \cdots, n,
$$


with the inner product

$$
\begin{aligned}
\langle u, v\rangle_{0}= & \sum_{m=0}^{n} \int_{\Omega_{m}}\left\{\left(\operatorname{curl} u_{1}, \operatorname{curl} v_{1}\right)+\left(\operatorname{curl} u_{2}, \operatorname{curl} v_{2}\right)\right. \\
& \left.+\left(B^{(m)} u_{1}, v_{1}\right)+\mu^{(m)}\left(u_{2}, v_{2}\right)\right\} d x
\end{aligned}
$$

From the results of [3] it follows that the expressions [v, $\left.u_{1}\right],\left[v, u_{2}\right],(v=$ $\left.v(x), x \in S, x \in S_{m}, m=1,2, \cdots, n\right)$ are well defined on $S, S_{m}$ and belong to $H_{-\frac{1}{2}}(S), H_{-\frac{1}{2}}\left(S_{m}\right)$.

This enables us to introduce in $\mathcal{H}_{0}$ the closed subspaces $\mathcal{H}_{1}, \widetilde{\mathcal{H}}_{1}$ :

$$
\begin{aligned}
\mathcal{H}_{1}= & \left\{u=\left\{u_{1}, u_{2}\right\} \in \mathcal{H}_{0}:\left[v, u_{1}^{(m-1)}\right]=\left[v, u_{1}^{(m)}\right],\left[v, u_{2}^{(m-1)}\right]\right. \\
& \left.=\left[v, u_{2}^{(m)}\right] \text { on } S_{m}, \quad m=1,2, \cdots, n ;\left[v, u_{1}\right]=0 \text { on } S\right\},
\end{aligned}
$$

the space $\widetilde{\mathcal{H}}_{1}$ is defined just as $\mathcal{H}_{1}$ with the only difference that $\left[v, u_{2}\right]$ vanishes on $S$.

We denote by $\mathcal{H}$ the real Hilbert space of quadruples $w=\left\{w_{1}, w_{2}, w_{3}, w_{4}\right\}$ of three-component vector-valued functions $w_{i}(x)$ such that

$$
w_{1}^{(m)} \in H_{1}\left(\Omega_{m}\right), w_{2}^{(m)}, w_{3}^{(m)}, w_{4}^{(m)} \in L_{2}\left(\Omega_{m}\right), \quad m=0,1, \cdots, n,
$$

where $w_{i}^{(m)}$ is the restriction of $w_{i}$ on $\Omega_{m}$. The inner product in $\mathcal{H}$ is given by

$$
\begin{aligned}
\langle v, w\rangle_{\mathcal{H}}= & \sum_{m=0}^{n} \int_{\Omega_{m}}\left\{\left(A_{i j}^{(m)} \frac{\partial v_{1}^{(m)}}{\partial x_{j}}, \frac{\partial w_{1}^{(m)}}{\partial x_{i}}\right)+\rho\left(v_{2}^{(m)}, w_{2}^{(m)}\right)\right. \\
& \left.+\left(B^{(m)} v_{3}^{(m)}, w_{3}^{(m)}\right)+\mu^{(m)}\left(v_{4}^{(m)}, w_{4}^{(m)}\right)\right\} d x+\int_{S} \beta\left(v_{1}, w_{1}\right) d S .
\end{aligned}
$$

The space $\widetilde{\mathcal{H}}$ is defined just as $\mathcal{H}$ with the only difference that the first vectorvalued function $w_{1}$ vanishes on $S$.

In $\mathcal{H}$ and $\widetilde{\mathcal{H}}$ we define unbounded operators $\mathcal{A}$ and $\widetilde{\mathcal{A}}$ :

$\mathcal{D}(\mathcal{A})$ consists of the elements $u=\left\{u_{1}, u_{2}, u_{3}, u_{4}\right\} \in \mathcal{H}$ such that

$$
\begin{aligned}
& A_{i j}^{(m)} \frac{\partial u_{1}^{(m)}}{\partial x_{j}}-A_{i}^{*} u_{3}^{(m)} \in H_{1}\left(\Omega_{m}\right), u_{2}^{(m)} \in H_{1}\left(\Omega_{m}\right),\left\{u_{3}, u_{4}\right\} \in \mathcal{H}_{1}, \\
& \left(A_{i j} \frac{\partial u_{1}}{\partial x_{j}}-A_{i}^{*} u_{3}\right) v_{i}+\beta u_{1}=0,\left[u_{3}, v\right]=0 \text { on } S,
\end{aligned}
$$




$$
\begin{aligned}
& \left\{\begin{array}{l}
u_{1}^{(m-1)}=u_{1}^{(m)}, \quad x \in S_{m}, \quad m=1,2, \cdots, n \\
\left(A_{i j}^{(m-1)} \frac{\partial u_{1}^{(m-1)}}{\partial x_{j}}-A_{i}^{*} u_{3}^{(m-1)}\right) v_{i}=\left(A_{i j}^{(m)} \frac{\partial u_{1}^{(m)}}{\partial x_{j}}-A_{i}^{*} u_{3}^{(m)}\right) v_{i} .
\end{array}\right. \\
& \mathcal{A} u=\left\{u_{2}, \rho^{-1} \frac{\partial}{\partial x_{i}}\left(A_{i j} \frac{\partial u_{1}}{\partial x_{j}}-A_{i}^{*} u_{3}\right),\right. \\
& \left.B^{-1}\left(\operatorname{curl} u_{4}-A_{i} \frac{\partial u_{2}}{\partial x_{i}}\right),-\mu^{-1} \operatorname{curl} u_{3}\right\}
\end{aligned}
$$

for $u=\left\{u_{1}, u_{2}, u_{3}, u_{4}\right\} \in \mathcal{D}(\mathcal{A})$.

The operator $\widetilde{\mathcal{A}}$ is defined just as $\mathcal{A}$ with the only difference that elements

$$
v=\left\{v_{1}, v_{2}, v_{3}, v_{4}\right\} \in \mathcal{D}(\widetilde{\mathcal{A}})\left(v \in \widetilde{\mathcal{H}},\left\{v_{3}, v_{4}\right\} \in \widetilde{\mathcal{H}}_{1}\right)
$$

satisfy another boundary conditions

$$
v_{1}=v_{2}=0, \quad\left[v, v_{4}\right]=0 \quad \text { on } S .
$$

The skew-selfadjointness of $\mathcal{A}$ and $\widetilde{\mathcal{A}}$ can be verified in the standard way.

Let $\mathcal{U}(t)$ and $\widetilde{\mathcal{U}}(t)$ be the strongly continuous groups of unitary operators generated by $\mathcal{A}$ and $\widetilde{\mathcal{A}}$.

We set

$$
\begin{aligned}
& M=\left\{w \in \mathcal{D}\left(\mathcal{A}^{*}\right): \mathcal{A}^{*} w=0\right\}, \\
& \tilde{M}=\left\{w \in \mathcal{D}\left(\widetilde{\mathcal{A}}^{*}\right): \widetilde{\mathcal{A}}^{*} w=0\right\} .
\end{aligned}
$$

Denote by $M_{1}$ and $\widetilde{M}_{1}$ the orthogonal complements of $M$ and $\tilde{M}$ in $\mathcal{H}$ and $\widetilde{\mathcal{H}}$ respectively.

Let us consider the problem (1.2)-(1.5) with homogeneous boundary conditions $\left(Q \equiv G \equiv 0\right.$ ). The kernel $M$ of $\mathcal{A}^{*}$ is nonempty, since it contains the quadruples $w=\left\{w_{1}, 0, \nabla g_{1}, \nabla g_{2}\right\}$, where $g_{1} \in H_{2}(\Omega), g_{2} \in H_{2}(\Omega) \cap \stackrel{\circ}{H}{ }_{1}(\Omega)$, $w_{1}$ is a solution of the following problem

$$
\frac{\partial}{\partial x_{i}}\left(A_{i j} \frac{\partial w_{1}}{\partial x_{j}}\right)=\frac{\partial}{\partial x_{i}}\left(A_{i}^{*} \nabla g_{1}\right), \quad x \in \Omega_{m}, \quad m=0,1, \cdots, n
$$




$$
\left\{\begin{array}{c}
w_{1}^{(m-1)}=w_{1}^{(m)}, \quad x \in S_{m}, \quad m=1,2, \cdots, n \\
\left(A_{i j}^{(m-1)} \frac{\partial w_{1}^{(m-1)}}{\partial x_{j}}\right) v_{i}=A_{i j}^{(m)} \frac{\partial w_{1}^{(m)}}{\partial x_{j}} v_{i}, \\
\left.\left(A_{i j} \frac{\partial w_{1}}{\partial x_{j}} v_{i}+\beta w_{1}\right)\right|_{s}=\left.A_{k}^{*} \nabla g_{1} v_{k}\right|_{s} .
\end{array}\right.
$$

It is obvious that $\mathcal{U}(t)$ takes $M_{1} \cap \mathcal{D}(\mathcal{A})$ into itself. Indeed, if $w \in M$ and $v \in M_{2} \cap \mathcal{D}(\mathcal{A})$, then

$$
\frac{d}{d t}\langle\mathcal{U}(t) v, w\rangle_{\mathcal{H}}=\langle\mathcal{A} \mathcal{U}(t) v, w\rangle_{\mathcal{H}}=\left\langle\mathcal{U}(t) v, \mathcal{A}^{*} w\right\rangle_{\mathcal{H}}=0 .
$$

We remark that element $v=\left\{v_{1}, v_{2}, v_{3}, v_{4}\right\} \in M_{1} \cap \mathcal{D}(\mathcal{A})$ possess the following property:

$$
\operatorname{div}\left\{B^{(m)} v_{3}^{(m)}+A_{k} \frac{\partial v_{1}^{(m)}}{\partial x_{k}}\right\}=0, \quad \operatorname{div} v_{4}^{(m)}=0, \quad m=0,1, \cdots, n
$$

in the sense of distributions.

Indeed, element $w=\left\{w_{1}, 0, \nabla g_{1}, 0\right\}$ where $g_{1} \in H_{2}(\Omega)$, supp $g_{1} \subset \Omega_{m}, w_{1}$ is a solution of the problem (2.1), belongs to $M$. Let $v \in M_{1} \cap \mathcal{D}(\mathcal{A})$. Then

$$
0=\langle v, w\rangle_{\mathcal{H}}=\int_{\Omega_{m}}\left(A_{k} \frac{\partial v_{1}^{(m)}}{\partial x_{k}}+B^{(m)} v_{3}^{(m)}, \nabla g_{1}\right) d x
$$

for an arbitrary $g_{1} \in H_{2}(\Omega)$, supp $g_{1} \subset \Omega_{m}$, which implies

$$
\operatorname{div}\left\{B^{(m)} v_{3}^{(m)}+A_{k} \frac{\partial v_{1}^{(m)}}{\partial x_{k}}\right\}=0
$$

in the sense of distributions.

It can be shown in a similar way (element $\left\{0,0,0, \nabla g_{2}\right\}$ belongs to $M$ for an arbitrary $g_{2} \in H_{2}(\Omega)$, supp $g_{2} \subset \Omega_{m}$ ) that $\operatorname{div} v_{4}^{(m)}=0$ in the sense of distributions.

Let us show that elements $v=\left\{v_{1}, v_{2}, v_{3}, v_{4}\right\} \in M_{1} \cap \mathcal{D}(\mathcal{A})$ satisfy the boundary condition

$$
\left.\left(v_{4}, v\right)\right|_{s}=0
$$


We note that element $w=\left\{0,0,0, \nabla g_{2}\right\}$ belongs to the kernel of $\mathcal{A}^{*}$ for an arbitrary $g_{2} \in H_{2}(\Omega), g_{2}=0$ in $\bar{\Omega}_{0} \cup \bar{\Omega}_{1} \cup \cdots \cup \bar{\Omega}_{n-1}$.

Thus, for $v=\left\{v_{1}, v_{2}, v_{3}, v_{4}\right\} \in M_{1} \cap \mathcal{D}(\mathcal{A})$ we have

$$
0=\langle v, w\rangle_{\mathcal{H}}=\int_{\Omega_{n}} \mu\left(v_{4}, \nabla g_{2}\right) d x=\int_{S} \mu g_{2}\left(v_{4}, v\right) d S,
$$

which implies (2.3).

Our next goal is to show that elements $v=\left\{v_{1}, v_{2}, v_{3}, v_{4}\right\} \in M_{1} \cap \mathcal{D}(\mathcal{A})$ satisfy the following interface conditions

$$
\left\{\begin{array}{l}
\mu^{(m-1)}\left(v_{4}^{(m-1)}, v\right)=\mu^{(m)}\left(v_{4}^{(m)}, v\right) \quad x \in S_{m}, \quad m=1,2, \cdots, n \\
\left(B^{(m-1)} v_{3}^{(m-1)}+A_{k} \frac{\partial v_{1}^{(m-1)}}{\partial x_{k}}, v\right)=\left(B^{(m)} v_{3}^{(m)}+A_{k} \frac{\partial v_{1}^{(m)}}{\partial x_{k}}, v\right) .
\end{array}\right.
$$

Since $w=\left\{0,0,0, \nabla g_{2}\right\}$ belongs to the kernel of $\widetilde{\mathcal{A}}^{*}$ for an arbitrary $g_{2} \in$ $H_{2}(\Omega) \cap \stackrel{\circ}{H}_{1}(\Omega)$, it follows that

$$
\begin{aligned}
0= & \langle v, w\rangle_{\mathcal{H}}=\sum_{m=0}^{n} \int_{\Omega_{m}} \mu^{(m)}\left(v_{4}^{(m)}, \nabla g_{2}\right) d x \\
& =\int_{S_{1}} \mu^{(0)}\left(v_{4}^{(0)}, v\right) g_{2} d S-\int_{S_{1}} \mu^{(1)}\left(v_{4}^{(1)}, v\right) g_{2} d S \\
& +\cdots+\int_{S_{n}} \mu^{(n-1)}\left(v_{4}^{(n-1)}, v\right) g_{2} d S-\int_{S_{n}} \mu^{(n)}\left(v_{4}^{(n)}, v\right) g_{2} d S .
\end{aligned}
$$

Now we choose $g_{2}$ such that $g_{2}=0$ on $S_{1}, \cdots, S_{m-1}, S_{m+1}, \cdots, S_{n}$. Then

$$
\int_{S_{m}}\left\{\mu^{(m-1)}\left(v_{4}^{(m-1)}, v\right)-\mu^{(m)}\left(v_{4}^{(m)}, v\right)\right\} g_{2} d S=0
$$

and we have

$$
\mu^{(m-1)}\left(v_{4}^{(m-1)}, v\right)=\mu^{(m)}\left(v_{4}^{(m)}, v\right) \text { on } S_{m}, m=1,2, \cdots, n .
$$

Moreover, element $w=\left\{w_{1}, 0, \nabla g_{1}, 0\right\}$ belongs to the kernel of $\mathcal{A}^{*}$ for an 
arbitrary $g_{1} \in \stackrel{\circ}{H}_{2}(\Omega)\left(w_{1}\right.$ is a solution of $\left.(2.1)\right)$. We have

$$
\begin{aligned}
0=\langle v, w\rangle_{\mathcal{H}}= & \sum_{m=0}^{n} \int_{\Omega_{m}}\left(A_{k} \frac{\partial v_{1}^{(m)}}{\partial x_{k}}+B^{(m)} v_{3}^{(m)}, \nabla g_{1}\right) d x \\
= & \sum_{m=1}^{n} \int_{S_{m}}\left\{\left(B^{(m-1)} v_{3}^{(m-1)}+A_{k} \frac{\partial v_{1}^{(m-1)}}{\partial x_{k}}, v\right)\right. \\
& \left.-\left(B^{(m)} v_{3}^{(m)}+A_{k} \frac{\partial v_{1}^{(m)}}{\partial x_{k}}, v\right)\right\} g_{1} d S .
\end{aligned}
$$

We choose $g_{1}$ such that $g_{1}=0$ on $S_{1}, \cdots, S_{m-1}, S_{m+1}, \cdots, S_{n}$. This gives us that

$$
\left(B^{(m-1)} v_{3}^{(m-1)}+A_{k} \frac{\partial v_{1}^{(m-1)}}{\partial x_{k}}, v\right)=\left(B^{(m)} v_{3}^{(m)}+A_{k} \frac{\partial v_{1}^{(m)}}{\partial x_{k}}, v\right) \text { on } S_{m} .
$$

Let us consider now the problem (1.6)-(1.9) with homogeneous boundary conditions $(\mathcal{D} \equiv \mathcal{P} \equiv 0)$. We remark that the kernel $\widetilde{M}$ of $\widetilde{\mathcal{A}}^{*}$ contains the quadruples $w=\left\{w_{1}, 0, \nabla g_{1}, \nabla g_{2}\right\}$, where $g_{1} \in H_{2}(\Omega), g_{2} \in H_{2}(\Omega) \cap \stackrel{\circ}{H_{1}}(\Omega)$, $w_{1}$ is a solution of (2.1) with the only difference that the functions $w_{1}$ satisfy the boundary condition:

$$
\left.w_{1}\right|_{s}=0
$$

It can be shown in the same way that elements $v=\left\{v_{1}, v_{2}, v_{3}, v_{4}\right\} \in \widetilde{M}_{1} \cap$ $\mathcal{D}(\widetilde{\mathcal{A}})$ satisfy $(2.2),(2.4)$.

Let us show that elements $v=\left\{v_{1}, v_{2}, v_{3}, v_{4}\right\} \in \widetilde{M}_{1} \cap \mathcal{D}(\widetilde{\mathcal{A}})$ satisfy the additional boundary condition

$$
\left.\left(B v_{3}+A_{k} \frac{\partial v_{1}}{\partial x_{k}}, v\right)\right|_{s}=0 .
$$

We remark that element $w=\left\{w_{1}, 0, \nabla g_{1}, 0\right\}$ belongs to the kernel of $\widetilde{\mathcal{A}}^{*}$ for an arbitrary $g_{1} \in H_{2}(\Omega), g_{1} \equiv 0$ in $\bar{\Omega}_{0} \cup \bar{\Omega}_{1} \cup \cdots \cup \bar{\Omega}_{n-1}, w_{1}$ is a solution of (2.1) with boundary condition (2.5). 
Thus, for $\left\{v_{1}, v_{2}, v_{3}, v_{4}\right\} \in \widetilde{M}_{1} \cap \mathcal{D}(\tilde{\mathcal{A}})$ we have

$$
\begin{aligned}
0=\langle v, w\rangle_{\widetilde{\mathcal{H}}} & =\int_{\Omega_{n}}\left(A_{k} \frac{\partial v_{1}}{\partial x_{k}}+B v_{3}^{(m)}, \nabla g_{1}\right) d x \\
& =\int_{S} g_{1}\left(B v_{3}+A_{k} \frac{\partial v_{1}}{\partial x_{k}}, v\right) d S
\end{aligned}
$$

which implies (2.6).

We arrive at the following assertion.

Theorem 2.1. Suppose that $f=\left\{f_{1}, f_{2}, f_{3}, f_{4}\right\} \in M_{1} \cap \mathcal{D}(\mathcal{A})(\varphi=$ $\left.\left\{\varphi_{1}, \varphi_{2}, \varphi_{3}, \varphi_{4}\right\} \in \tilde{M}_{1} \cap \mathcal{D}(\tilde{\mathcal{A}})\right)$. Then there exists a unique solution $\{u(x, t), E(x, t), H(x, t)\}(v(x, t), \Phi(x, t), \Psi(x, t))$ of (1.2)-(1.5) ((1.6)(1.9)) with zero boundary conditions such that for all $t \geq 0$

$$
\begin{aligned}
& u(x, t) \in H_{2}\left(\Omega_{m}\right), \frac{\partial u}{\partial t}(x, t), E(x, t), H(x, t) \in H_{1}\left(\Omega_{m}\right), \frac{\partial^{2} u}{\partial t^{2}}(x, t) \in L_{2}\left(\Omega_{m}\right) \\
& \left(v(x, t) \in H_{2}\left(\Omega_{m}\right), \frac{\partial v}{\partial t}(x, t), \Phi(x, t), \Psi(x, t) \in H_{1}\left(\Omega_{m}\right), \frac{\partial^{2} v}{\partial t^{2}}(x, t) \in L_{2}\left(\Omega_{m}\right)\right), \\
& m=0,1, \cdots, n ; \\
& (H, v)=0\left(\left(B \Phi+A_{k} \frac{\partial v}{\partial x_{k}}, v\right)=0\right), \quad x \in S, \quad t \geq 0 .
\end{aligned}
$$

Moreover, $\{u, E, H\}(\{v, \Phi, \Psi\})$ satisfies the additional interface conditions (2.4), where

$$
v_{1}=u, \quad v_{3}=E, \quad v_{4}=H \quad\left(v_{1}=v, v_{3}=\Phi, v_{4}=\Psi\right)
$$

and

$$
\left\|\left\{u, \frac{\partial u}{\partial t}, E, H\right\}\right\|_{\mathcal{H}}=\|f\|_{\mathcal{H}}\left(\left\|\left\{v, \frac{\partial v}{\partial t}, \Phi, \Psi\right\}\right\| \widetilde{\mathcal{H}}=\|\varphi\|_{\widetilde{\mathcal{H}}}\right) .
$$

Let $f=\left\{f_{1}, f_{2}, f_{3}, f_{4}\right\} \in \mathcal{H}$ and $f^{n}=\left\{f_{1}^{n}, f_{2}^{n}, f_{3}^{n}, f_{4}^{n}\right\} \in \mathcal{D}(\mathcal{A})$, such that $\left\|f-f^{n}\right\|_{\mathcal{H}} \rightarrow 0$ as $n \rightarrow \infty$. Then, $\mathcal{U}(t) f^{n}$ satisfies the following identity

$$
\int_{0}^{T}\left\{\left\langle\mathcal{U}(t) f^{n}, \frac{d V}{d t}\right\rangle_{\mathcal{H}}+\left\langle\mathcal{U}(t) f^{n}, \mathcal{A}^{*} V\right\rangle_{\mathcal{H}}\right\} d t=-\left\langle f^{n}, V(0)\right\rangle_{\mathcal{H}},
$$


where $V(t) \in L_{2}\left(0, T ; \mathcal{D}\left(\mathcal{A}^{*}\right)\right), V_{t}(t) \in L_{2}(0, T ; \mathcal{H}), V(T)=0$.

From this we easily obtain that

$$
\int_{0}^{T}\left\{\left\langle\mathcal{U}(t) f, \frac{d V}{d t}\right\rangle_{\mathcal{H}}+\left\langle\mathcal{U}(t) f, \mathcal{A}^{*} V\right\rangle_{\mathcal{H}}\right\} d t=-\langle f, V(0)\rangle_{\mathcal{H}},
$$

i.e., $\mathcal{U}(t) f$ is the weak solution of the abstract Cauchy problem

$$
\frac{d u}{d t}=\mathcal{A} u,\left.\quad u\right|_{t=0}=f .
$$

We note that $\mathcal{U}(t)$ takes $M_{1}$ into itself. Indeed, if $g \in M$ and $V(t)=(T-t) g$, then from (2.7) it follows that

$$
\int_{0}^{T}\langle\mathcal{U}(t) f, g\rangle_{\mathcal{H}} d t=T\langle f, g\rangle_{\mathcal{H}}
$$

Thus,

$$
\langle\mathcal{U}(t) f, g\rangle_{\mathcal{H}}=\langle f, g\rangle_{\mathcal{H}} \text { for } t \geq 0 .
$$

In the same way we get the corresponding properties for $\widetilde{\mathcal{U}}(t)$.

Let us now concern ourselves with the simultaneous boundary observability for a pair of piezoelectric systems. The proof is based on the invariance of the piezoelectric system relative to the one-parameter group of dilations in all variables. This property of the system leads to the following identity:

$$
\begin{aligned}
& 2\left(t \frac{\partial u}{\partial t}+(\nabla g, \nabla) u+u, \rho \frac{\partial^{2} u}{\partial t^{2}}-\frac{\partial}{\partial x_{i}}\left\{A_{i j} \frac{\partial u}{\partial x_{j}}-A_{i}^{*} E\right\}\right) \\
& +2\left(t E+\mu[\nabla g, H], \frac{\partial}{\partial t}\left\{B E+A_{i} \frac{\partial u}{\partial x_{i}}\right\}-\operatorname{curl} H\right) \\
& +2\left(t H-\left[\nabla g, B E+A_{k} \frac{\partial u}{\partial x_{k}}\right], \mu \frac{\partial H}{\partial t}+\operatorname{curl} E\right) \\
& +2(E, \nabla g) \operatorname{div}\left\{B E+A_{k} \frac{\partial u}{\partial x_{k}}\right\}+2 \mu(H, \nabla g) \operatorname{div} H \\
& =\frac{\partial}{\partial t}\left\{t\left[\rho\left|\frac{\partial u}{\partial t}\right|^{2}+\left(A_{i j} \frac{\partial u}{\partial x_{j}} \frac{\partial u}{\partial x_{i}}\right)+(B E, E)+\mu|H|^{2}\right]\right. \\
& \left.+2 \rho\left(\frac{\partial u}{\partial t},(\nabla g, \nabla) u+u\right)+2 \mu\left([\nabla g, H], B E+A_{k} \frac{\partial u}{\partial x_{k}}\right)\right\}
\end{aligned}
$$




$$
\begin{aligned}
& -\frac{\partial}{\partial x_{i}}\left\{2\left(t \frac{\partial u}{\partial t}+(\nabla g, \nabla) u+u, A_{i j} \frac{\partial u}{\partial x_{j}}-A_{i}^{*} E\right)\right. \\
& \left.+\frac{\partial g}{\partial x_{i}}\left[\rho\left|\frac{\partial u}{\partial t}\right|^{2}-\left(A_{p q} \frac{\partial u}{\partial x_{q}}, \frac{\partial u}{\partial x_{p}}\right)\right]\right\} \\
& -\operatorname{div}\left\{2 t[H, E]+\nabla g(B E, E)+\nabla g \mu|H|^{2}-2 B E(E, \nabla g)\right. \\
& \left.-2 \mu H(H, \nabla g)+2\left[E\left[\nabla g, A_{k} \frac{\partial u}{\partial x_{k}}\right]\right]\right\} \\
& -\left\{(\Delta g-1)\left(A_{i j} \frac{\partial u}{\partial x_{j}}, \frac{\partial u}{\partial x_{i}}\right)-2 \frac{\partial^{2} g}{\partial x_{p} \partial x_{i}}\left(A_{i j} \frac{\partial u}{\partial x_{j}}, \frac{\partial u}{\partial x_{p}}\right)+(3-\Delta g) \rho\left|\frac{\partial u}{\partial t}\right|^{2}\right\} \\
& -\left\{2 \frac{\partial^{2} g}{\partial x_{i} \partial x_{k}} b_{i j} E^{j} E^{k}-(\Delta g-1)(B E, E)+2 \frac{\partial^{2} g}{\partial x_{i} \partial x_{j}} \mu H^{i} H^{j}-(\Delta g-1) \mu|H|^{2}\right\} \\
& -2\left(E, \frac{\partial^{2} g}{\partial x_{i} \partial x_{k}} A_{k} \frac{\partial u}{\partial x_{i}}+\left(A_{k} \frac{\partial u}{\partial x_{k}}, \nabla\right) \nabla g-(\Delta g-1) A_{k} \frac{\partial u}{\partial x_{k}}\right),
\end{aligned}
$$

where $g(x)$ is an arbitrary smooth function, $\nabla=\left(\frac{\partial}{\partial x_{1}}, \frac{\partial}{\partial x_{2}}, \frac{\partial}{\partial x_{3}}\right)$. For $g(x)=$ $2^{-1}\left|x-x^{0}\right|^{2},(2.8)$ represents a conservation law.

Let $f=\left\{f_{1}, f_{2}, f_{3}, f_{4}\right\} \in M_{1} \cap \mathcal{D}(\mathcal{A})$ and $\{u(x, t), E(x, t), H(x, t)\}$ is the corresponding solution of (1.2)-(1.5) with zero boundary conditions.

From (2.8) after integration over $\Omega_{m} \times(0, T)$ and summation over $m$ we get

$$
\begin{aligned}
& T \sum_{m=0}^{n} \int_{\Omega_{m}}\left\{\rho\left|\frac{\partial u}{\partial t}\right|^{2}+\left(A_{i j} \frac{\partial u}{\partial x_{j}}, \frac{\partial u}{\partial x_{i}}\right)+(B E, E)+\mu|H|^{2}\right\} d x \\
& \quad+2 \sum_{m=0}^{n} \int_{\Omega_{m}}\left\{\rho\left(\frac{\partial u}{\partial t},(\nabla g, \nabla) u+u\right)\right. \\
& \left.\quad+\mu\left([\nabla g, H], B E+A_{k} \frac{\partial u}{\partial x_{k}}\right)\right\}\left.d x\right|_{t=0} ^{t=T} \\
& \quad=\sum_{m=1}^{n} \int_{0}^{T} \int_{S_{m}}\left\{\mathcal{B}_{m-1}(u, E, H)-\mathcal{B}_{m}(u, E, H)\right\} d S d t \\
& \quad+\int_{0}^{T} \int_{S} \mathcal{B}_{n}(u, E, H) d S d t+\sum_{m=1}^{n} \int_{0}^{T} \int_{\Omega_{m}} \mathcal{F}_{m}(u, E, H ; g) d x d t,
\end{aligned}
$$

where $\mathcal{F}_{m}(u, E, H ; g)$ is the restriction of the last three terms on the right-hand 
side of (2.8) on $\Omega_{m}$ and

$$
\begin{aligned}
& \mathcal{B}_{m}(u, E, H)=2\left(t \frac{\partial u^{(m)}}{\partial t}+(\nabla g, \nabla) u^{(m)}\right. \\
& \left.+u^{(m)}, A_{i j}^{(m)} \frac{\partial u^{(m)}}{\partial x_{j}} v_{i}-A_{i}^{*} E^{(m)} v_{i}\right) \\
& +\frac{\partial g}{\partial v}\left[\rho\left|\frac{\partial u^{(m)}}{\partial t}\right|^{2}-\left(A_{p q}^{(m)} \frac{\partial u^{(m)}}{\partial x_{q}}, \frac{\partial u^{(m)}}{\partial x_{p}}\right)\right] \\
& +2 t\left(v,\left[H^{(m)}, E^{(m)}\right]\right)+\frac{\partial g}{\partial v}\left(B^{(m)} E^{(m)}, E^{(m)}\right) \\
& +\frac{\partial g}{\partial v} \mu^{(m)}\left|H^{(m)}\right|^{2}-2\left(B^{(m)} E^{(m)}, v\right)\left(E^{(m)}, \nabla g\right) \\
& \quad-2 \mu^{(m)}\left(H^{(m)}, v\right)\left(H^{(m)}, \nabla g\right)+2\left(\left[\nabla g, A_{k} \frac{\partial u^{(m)}}{\partial x_{k}}\right],\left[v, E^{(m)}\right]\right) .
\end{aligned}
$$

The next assertion is of a technical nature and can be proved by direct computations.

Lemma 2.2. The following representation holds:

$$
\begin{aligned}
\mathcal{B}_{m-1} & (u, E, H)-\mathcal{B}_{m}(u, E, H) \\
& =-\frac{\partial g}{\partial v}\left\{\left(\left(A_{i j}^{(m-1)}-A_{i j}^{(m)}\right) \frac{\partial u^{(m-1)}}{\partial x_{j}}, \frac{\partial u^{(m-1)}}{\partial x_{i}}\right)\right. \\
& +\left(A_{i j}^{(m)}\left(\frac{\partial u^{(m)}}{\partial x_{j}}-\frac{\partial u^{(m-1)}}{\partial x_{j}}\right),\left(\frac{\partial u^{(m)}}{\partial x_{i}}-\frac{\partial u^{(m-1)}}{\partial x_{i}}\right)\right) \\
& +\left(\mu^{(m)}-\mu^{(m-1)}\right)\left(\left|\left[H^{(m)}, v\right]\right|^{2}+\frac{\mu^{(m)}}{\mu^{(m-1)}}\left|\left(H^{(m)}, v\right)\right|^{2}\right) \\
& +\left(\left(B^{(m)}-B^{(m-1)}\right) E^{(m)}, E^{(m)}\right) \\
& \left.+\left(B^{(m-1)}\left(E^{(m)}-E^{(m-1)}\right), E^{(m)}-E^{(m-1)}\right)\right\} .
\end{aligned}
$$

Let us now concern ourselves with an estimate of the integral of $\mathcal{F}_{m}(u, E, H ; g)$ over $\Omega_{m} \times(0, T)$.

We consider the elliptic problem

$$
\Delta W=1 \quad \text { on } \quad \Omega,\left.\quad \frac{\partial W}{\partial v}\right|_{s}=\frac{\operatorname{mes} \Omega}{\operatorname{mes} S},
$$


which admits a solution $W(x) \in C^{2}(\Omega) \cap C^{1}(\bar{\Omega})$.

We set

$$
g(x)=\delta W(x)+\frac{1}{2}\left|x-x^{0}\right|^{2}, \quad \delta>0 .
$$

Direct computations give us that (the index $m$ is omitted for simplicity of notations)

$$
\begin{gathered}
\mathcal{F}_{m}(u, E, H ; g)=\delta\left(A_{i j} \frac{\partial u}{\partial x_{j}}, \frac{\partial u}{\partial x_{i}}\right)-2 \delta \frac{\partial^{2} W}{\partial x_{p} \partial x_{i}}\left(A_{i j} \frac{\partial u}{\partial x_{j}}, \frac{\partial u}{\partial x_{p}}\right)-\delta \rho\left|\frac{\partial u}{\partial t}\right|^{2} \\
+2 \delta \frac{\partial^{2} W}{\partial x_{i} \partial x_{k}} b_{i j} E^{j} E^{k}+2 \delta \frac{\partial^{2} W}{\partial x_{i} \partial x_{j}} \mu H^{i} H^{j}-\delta\left((B E, E)+\mu|H|^{2}\right) \\
+2 \delta\left(E, \frac{\partial^{2} W}{\partial x_{i} \partial x_{k}} A_{k} \frac{\partial u}{\partial x_{i}}+\left(A_{k} \frac{\partial u}{\partial x_{k}}, \nabla\right) \nabla W-A_{k} \frac{\partial u}{\partial x_{k}}\right)
\end{gathered}
$$

We have

$$
\begin{aligned}
- & 2 \delta \frac{\partial^{2} W}{\partial x_{p} \partial x_{i}}\left(A_{i j} \frac{\partial u}{\partial x_{j}}, \frac{\partial u}{\partial x_{p}}\right) \\
\leq & \delta\left[\sigma_{1}+\frac{27}{C_{0} \sigma_{1}} C(A) C^{2}(W)\right]\left(A_{i j} \frac{\partial u}{\partial x_{j}}, \frac{\partial u}{\partial x_{i}}\right),
\end{aligned}
$$

where $\sigma_{1}>0, C_{0}$ is such that

$$
\sum_{i, j=1}^{3}\left(A_{i j} \eta_{j}, \eta_{i}\right) \geq C_{0} \sum_{i=1}^{3}\left|\eta_{i}\right|^{2}, \quad \eta_{i} \in \mathbb{R}^{3},
$$

and

$$
C(A)=\max _{x \in \bar{\Omega} i, j=1,2,3}\left\|A_{i j}(x)\right\|, \quad C(W)=\max _{x \in \bar{\Omega} i, j=1,2,3}\left|\frac{\partial^{2} W(x)}{\partial x_{i} \partial x_{j}}\right| .
$$

We note that $C(W) \geq 1 / 3$.

Next, we get the estimate

$$
\begin{aligned}
& 2 \delta\left(E, \frac{\partial^{2} W}{\partial x_{i} \partial x_{k}} A_{k} \frac{\partial u}{\partial x_{i}}+\left(A_{k} \frac{\partial u}{\partial x_{k}}, \nabla\right) \nabla W-A_{k} \frac{\partial u}{\partial x_{k}}\right) \\
& \quad \leq \delta \sigma_{2}(1+2 C(W)) C_{1}(A)|E|^{2} \\
& \quad+\delta \frac{1}{\sigma_{2}}(1+2 C(W)) C_{1}(A) \frac{1}{C_{0}}\left(A_{i j} \frac{\partial u}{\partial x_{j}}, \frac{\partial u}{\partial x_{i}}\right),
\end{aligned}
$$


where $\sigma_{2}$ is an arbitrary positive number,

$$
C_{1}(A)=\max _{k=1,2,3}\left\|A_{k}\right\|
$$

We have

$$
\begin{aligned}
& 2 \delta \frac{\partial^{2} W}{\partial x_{i} \partial x_{k}} b_{i j} E^{j} E^{k}+2 \delta \frac{\partial^{2} W}{\partial x_{i} \partial x_{j}} \mu H^{i} H^{j} \\
& \quad \leq \delta 6 C(W) \mu|H|^{2}+\delta 6 C(W)\|B\| \frac{1}{b_{0}}(B E, E) .
\end{aligned}
$$

Thus, from (2.12)-(2.14) we get the estimate

$$
\begin{aligned}
& \mathcal{F}_{m}(u, E, H ; g) \leq \delta\left[1+\sigma_{1}+\frac{27}{C_{0} \sigma_{1}} C(A) C^{2}(W)\right. \\
& \left.+\frac{1}{C_{0} \sigma_{2}} C_{1}(A)(1+2 C(W))\right]\left(A_{i j} \frac{\partial u}{\partial x_{j}}, \frac{\partial u}{\partial x_{i}}\right)+\delta[6 C(W)-1] \mu|H|^{2} \\
& +\delta\left[6 C(W) \frac{1}{b_{0}}\|B\|+\sigma_{2}(1+2 C(W)) C_{1}(A) \frac{1}{b_{0}}\right](B E, E) .
\end{aligned}
$$

We now choose $\sigma_{1}$ and $\sigma_{2}$. We set

$$
\sigma_{1}=C(W) \sqrt{\frac{C(A)}{C_{0}}}, \quad \sigma_{2}=\sqrt{\frac{b_{0}}{C_{0}}} .
$$

From the inequality (2.15) it follows that

$$
\begin{aligned}
& \sum_{m=0}^{n} \int_{0}^{T} \int_{\Omega_{m}} \mathcal{F}_{m}(u, E, H ; g) d x d t \leq \delta C_{1} \sum_{m=0}^{n} \int_{0}^{T} \int_{\Omega_{m}} \\
& \quad\left\{\left(A_{i j}^{(m)} \frac{\partial u^{(m)}}{\partial x_{j}}, \frac{\partial u^{(m)}}{\partial x_{i}}\right)+\left(B^{(m)} E^{(m)}, E^{(m)}\right)+\mu^{(m)}\left|H^{(m)}\right|^{2}\right\} d x d t \\
& \quad \leq \delta C_{1} T\left\|\left\{u, \frac{\partial u}{\partial t}, E, H\right\}\right\|_{\mathcal{H}}^{2},
\end{aligned}
$$

where $\delta$ is an arbitrary positive number and

$$
C_{1}=\frac{C_{1}(A)(1+2 C(W))}{\sqrt{C_{0} b_{0}}}+\max \left\{1+28 C(W) \sqrt{\frac{C(A)}{C_{0}}}, 6 C(W)\|B\| \frac{1}{b_{0}}\right\} .
$$

From here on we will assume that $\Omega$ and $S_{m}$ satisfy the following conditions: there exists $\delta_{1} \geq 0$ such that 
(i) $\delta_{1} C_{1}<1$,

(ii) $\delta_{1} \frac{\partial W}{\partial v}+\left(x-x^{0}, v\right) \geq 0$ for some point $x^{0} \in \Omega, x \in S_{m}, m=1,2, \cdots, n$, (iii) $\delta_{1} \frac{\text { mes } \Omega}{\text { mes } S}+\left(x-x^{0}, v\right)>0, x \in S, x^{0}$ is defined in (ii).

We note that the above conditions are valid when $\delta_{1}=0$ for star-shaped surfaces $S_{1}, S_{2}, \cdots, S_{n}$, and strongly star-shaped surface $S$, i.e.,

$$
\left(x-x^{0}, v\right)>0, \quad x \in S .
$$

Moreover, if $S_{1}, S_{2}, \cdots, S_{n}$ are strongly star-shaped with respect to a point $x^{0} \in \Omega$, then, the above conditions hold with $\delta_{1}>0$ for a class of domains which includes star-shaped domains.

Henceforth we set

$$
g(x)=\delta_{1} W(x)+\frac{1}{2}\left|x-x^{0}\right|^{2},
$$

where $\delta_{1}$ is defined in (2.17).

Our next goal is to estimate the second integral on the left-hand side of (2.9). The following inequality is proved by standard arguments

$$
\begin{aligned}
& 2 \sum_{m=0}^{n} \int_{\Omega_{m}}\left\{\rho\left(\frac{\partial u}{\partial t},(\nabla \varphi, \nabla) u+u\right)\right. \\
& \left.\quad+\mu\left([\nabla \varphi, H], B E+A_{k} \frac{\partial u}{\partial x_{k}}\right)\right\} d x \leq C_{2}\left\|\left\{u, \frac{\partial u}{\partial t}, E, H\right\}\right\|_{\mathcal{H}}^{2},
\end{aligned}
$$

where

$$
\begin{aligned}
& C_{2}=\max \left\{2, \frac{1+C(\Omega)}{c_{2}}\left(\rho+\mu_{1} C_{1}(A)\right), 2 C(\Omega), \frac{\mu_{1} b_{1}}{b_{0}} C(\Omega)\right\}, \\
& C(\Omega)=\max _{x \in \bar{\Omega}}\left\{\left|x-x^{0}\right|+\delta_{1}|\nabla W(x)|\right\}, \quad \mu_{1}=\max _{\bar{\Omega}} \mu, \quad b_{1}=\max _{x \in \bar{\Omega}}\|B(x)\|,
\end{aligned}
$$

$c_{2}>0$ is such that

$$
c_{2} \sum_{m=0}^{n}\left\|u^{(m)}\right\|_{H_{1}\left(\Omega_{m}\right)}^{2} \leq \sum_{m=0}^{n} \int_{\Omega_{m}}\left(A_{i j}^{(m)} \frac{\partial u^{(m)}}{\partial x_{j}}, \frac{\partial u^{(m)}}{\partial x_{i}}\right) d x+\int_{S} \beta\left|u^{(n)}\right|^{2} d S,
$$


$u^{(m)} \in H_{2}\left(\Omega_{m}\right), u^{(m-1)}=u^{(m)}$ on $S_{m}, m=1,2, \cdots, n$.

Now, we are concern with an estimate of the surface integral (over $S \times(0, T)$ ) in (2.9).

Using the boundary conditions (1.5) $(Q \equiv G \equiv 0)$ and additional boundary condition

$$
\left.(H, v)\right|_{S \times(0, T)}=0,
$$

we get

$$
\begin{aligned}
& \int_{0}^{T} \int_{S} \mathcal{B}_{n}(u, E, H) d S d t=-T \int_{S} \beta|u(x, T)|^{2} d S \\
& +\int_{0}^{T} \int_{S}\left\{\frac{\partial g}{\partial v}\left(\rho\left|\frac{\partial u}{\partial t}\right|^{2}+\mu|[H, v]|^{2}\right)\right. \\
& +\beta|(\nabla g, \nabla) u|^{2}-\frac{\partial g}{\partial v}\left(A_{i j} \frac{\partial u}{\partial x_{j}}, \frac{\partial u}{\partial x_{i}}\right) \\
& \left.\quad-\frac{\partial g}{\partial v}(B v, v)|(E, v)|^{2}-\beta|u+(\nabla g, \nabla) u|^{2}\right\} d S d t .
\end{aligned}
$$

Let $\chi>0$ be such that

$$
\frac{\partial g}{\partial v} \geq \chi|\nabla g|, \quad x \in S .
$$

We have

$$
\beta|(\nabla g, \nabla) u|^{2} \leq \beta \frac{1}{C_{0}}|\nabla g|^{2}\left(A_{i j} \frac{\partial u}{\partial x_{j}}, \frac{\partial u}{\partial x_{i}}\right) .
$$

Assume that $\beta=\beta(x)$ satisfies the following condition

$$
\beta(x) \leq \frac{C_{0} \chi}{C(\Omega)} .
$$

Thus, from (2.20) we obtain the inequality

$$
\beta|(\nabla g, \nabla) u|^{2} \leq \chi|\nabla g|\left(A_{i j} \frac{\partial u}{\partial x_{j}}, \frac{\partial u}{\partial x_{i}}\right) \leq \frac{\partial g}{\partial v}\left(A_{i j} \frac{\partial u}{\partial x_{j}}, \frac{\partial u}{\partial x_{i}}\right),
$$


from which it follows that

$$
\begin{aligned}
& \int_{0}^{T} \int_{S} \mathcal{B}_{n}(u, E, H) d S d t \\
& \leq \int_{0}^{T} \int_{S} \frac{\partial g}{\partial v}\left\{\rho\left|\frac{\partial u}{\partial t}\right|^{2}+\mu|[H, v]|^{2}\right\} d S d t-T \int_{S} \beta|u(x, T)|^{2} d S .
\end{aligned}
$$

Suppose that the coefficients of the systems (1.2) satisfy the following monotonicity conditions

$$
\left\{\begin{array}{l}
\left(\left(A_{i j}^{(m-1)}-A_{i j}^{(m)}\right) \eta_{j}, \eta_{i}\right) \geq 0, \quad \eta_{i} \in \mathbb{R}^{3}, \\
\left(\left(B^{(m)}-B^{(m-1)}\right) \eta, \eta\right) \geq 0, \quad \eta \in \mathbb{R}^{3}, \quad m=1,2, \cdots, n \\
\mu^{(m)} \geq \mu^{(m-1)}
\end{array}\right.
$$

Using these conditions and Lemma 2.2, we obtain

$$
\mathcal{B}_{m-1}(u, E, H)-\mathcal{B}_{m}(u, E, H) \leq 0, \quad m=1,2, \cdots, n .
$$

Thus, from the identity (2.9) and the inequalities (2.16), (2.18), (2.21), (2.23) we get

$$
\begin{aligned}
& \left(1-\delta_{1} C_{1}\right)\left(T-T_{1}\right) \\
& \left\{\sum_{m=0}^{n} \int_{\Omega_{m}}\left[\rho\left|\frac{\partial u}{\partial t}\right|^{2}+\left(A_{i j} \frac{\partial u}{\partial x_{j}}, \frac{\partial u}{\partial x_{i}}\right)+(B E, E)+\mu|H|^{2}\right]\right. \\
& \left.+\int_{S} \beta|u(x, T)|^{2} d S\right\} \leq \int_{0}^{T} \int_{S} \frac{\partial g}{\partial v}\left\{\rho\left|\frac{\partial u}{\partial t}\right|^{2}+\mu|[H, v]|^{2}\right\} d S d t
\end{aligned}
$$

where

$$
T_{1}=\frac{2 C_{2}}{1-\delta_{1} C_{1}} .
$$

We now consider the problem (1.6)-(1.9) with zero boundary conditions. Let $\varphi=\left\{\varphi_{1}, \varphi_{2}, \varphi_{3}, \varphi_{4}\right\} \in \widetilde{M}_{1} \cap \mathcal{D}(\widetilde{\mathcal{A}})$ and $\left\{v, \frac{\partial v}{\partial t}, \Phi, \Psi\right\}=\widetilde{\mathcal{U}}(t) \varphi$.

In this case we have

$$
\begin{aligned}
\int_{0}^{T} & \int_{S} \mathcal{B}_{n}(v, \Phi, \Psi) d S d t \\
\quad= & \int_{0}^{T} \int_{S} \frac{\partial g}{\partial v}\left\{\left(A_{i j} \frac{\partial v}{\partial x_{j}}, \frac{\partial v}{\partial x_{i}}\right)+(B \Phi, \Phi)-\mu|(\Psi, v)|^{2}\right\} d S d t
\end{aligned}
$$


In the same way we get the estimate

$$
\begin{aligned}
& \left(1-\delta_{1} C_{1}\right)\left(T-T_{1}\right) \\
& \sum_{m=0}^{n} \int_{\Omega_{m}}\left\{\rho\left|\frac{\partial v}{\partial t}\right|^{2}+\left(A_{i j} \frac{\partial v}{\partial x_{j}}, \frac{\partial v}{\partial x_{i}}\right)+(B \Phi, \Phi)+\mu|\Psi|^{2}\right\} d x \\
& \leq \int_{0}^{T} \int_{S} \frac{\partial g}{\partial v}\left\{\left(A_{i j} \frac{\partial v}{\partial x_{j}}, \frac{\partial v}{\partial x_{i}}\right)+(B \Phi, \Phi)\right\} d S d t .
\end{aligned}
$$

Our next goal is to obtain the simultaneous boundary observation for a pair of systems (1.2), (1.6).

Let $f \in M_{1} \cap \mathcal{D}(\mathcal{A}), \varphi \in \widetilde{M}_{1} \cap \mathcal{D}(\widetilde{\mathcal{A}})$ and

$$
\left\{u, \frac{\partial u}{\partial t}, E, H\right\}=\mathcal{U}(t) f, \quad\left\{v, \frac{\partial v}{\partial t}, \Phi, \Psi\right\}=\widetilde{U}(t) \varphi .
$$

We can immediately verify the identity

$$
\begin{gathered}
\left.\sum_{m=0}^{n} \int_{\Omega_{m}}\left\{\rho\left(\frac{\partial u}{\partial t}, \frac{\partial v}{\partial t}\right)+\left(A_{i j} \frac{\partial u}{\partial x_{j}}, \frac{\partial v}{\partial x_{i}}\right)+(B E, \Phi)+\mu(H, \Psi)\right\} d x\right|_{t=0} ^{t=T} \\
=\int_{0}^{T} \int_{S}\left\{\left(\frac{\partial u}{\partial t},\left(A_{i j} \frac{\partial v}{\partial x_{j}}-A_{i}^{*} \Phi\right) v_{i}\right)+(\Phi,[v, H])\right\} d S d t
\end{gathered}
$$

The following formula can be proved by direct computations:

$$
\begin{aligned}
& \left(\frac{\partial u}{\partial t},\left(A_{i j} \frac{\partial v}{\partial x_{j}}-A_{i}^{*} \Phi\right) v_{i}\right)+(\Phi,[v, H]) \\
& \quad=\frac{1}{2}\left|\frac{\partial u}{\partial t}+\left(A_{i j} \frac{\partial v}{\partial x_{j}}-A_{i}^{*} \Phi\right) v_{i}\right|^{2}+\frac{1}{2}|\Phi-[H, v]|^{2} \\
& \quad-\frac{1}{2}\left|\frac{\partial u}{\partial t}\right|^{2}-\frac{1}{2}\left|A_{i j} \frac{\partial v}{\partial x_{j}} v_{i}\right|^{2}-\frac{1}{2}\left|A_{i}^{*} \Phi v_{i}\right|^{2}-\frac{1}{2}|\Phi|^{2} \\
& \quad-\frac{1}{2}|[H, v]|^{2}+\left(A_{i j} \frac{\partial v}{\partial x_{j}} v_{i}, A_{k}^{*} \Phi v_{k}\right) .
\end{aligned}
$$

We have

$$
\begin{gathered}
\left(A_{i j} \frac{\partial v}{\partial x_{j}} v_{i}, A_{k}^{*} \Phi v_{k}\right) \leq \frac{1}{2} \frac{1}{1+\varepsilon}\left|A_{i j} \frac{\partial v}{\partial x_{j}} v_{i}\right|^{2}+\frac{1}{2}(1+\varepsilon)\left|A_{k}^{*} \Phi v_{k}\right|^{2}, \\
\left|A_{k}^{*} \Phi v_{k}\right|^{2} \leq 9 C_{1}^{2}(A)|\Phi|^{2} .
\end{gathered}
$$


We set

$$
\varepsilon=\frac{1-\delta_{0}}{9 C_{1}^{2}(A)}, \quad 0<\delta_{0}<1 .
$$

Then (2.27) implies the inequality

$$
\begin{aligned}
& \left(\frac{\partial u}{\partial t},\left(A_{i j} \frac{\partial v}{\partial x_{j}}-A_{i}^{*} \Phi\right) v_{i}\right)+(\Phi,[v, H]) \\
& \quad \leq \frac{1}{2}\left|\frac{\partial u}{\partial t}+\left(A_{i j} \frac{\partial v}{\partial x_{j}}-A_{i}^{*} \Phi\right) v_{i}\right|^{2}+\frac{1}{2}|\Phi-[H, v]|^{2}-\frac{1}{2}\left|\frac{\partial u}{\partial t}\right|^{2} \\
& \quad-\frac{1}{2}|[H, v]|^{2}-\frac{1-\delta_{0}}{2\left(9 C_{1}^{2}(A)+1-\delta_{0}\right)}\left|A_{i j} \frac{\partial v}{\partial x_{j}} v_{i}\right|^{2}-\frac{\delta_{0}}{2}|\Phi|^{2} .
\end{aligned}
$$

Henceforth we assume that matrices $A_{i j}$ satisfy the following condition

$$
\left|A_{i j}^{(n)} v_{j} v_{i} \xi\right| \geq a_{0}|\xi|, \quad \xi \in \mathbb{R}^{3} .
$$

Taking (2.29) into account, from (2.28) we find that

$$
\begin{aligned}
& \left(\frac{\partial u}{\partial t},\left(A_{i j} \frac{\partial v}{\partial x_{j}}-A_{i}^{*} \Phi\right) v_{i}\right)+(\Phi,[v, H]) \\
& \quad \leq \frac{1}{2}\left|\frac{\partial u}{\partial t}+\left(A_{i j} \frac{\partial v}{\partial x_{j}}-A_{i}^{*} \Phi\right) v_{i}\right|^{2}+\frac{1}{2}|\Phi-[H, v]|^{2} \\
& \quad-\frac{1}{2}\left|\frac{\partial u}{\partial t}\right|^{2}-\frac{1}{2}|[H, v]|^{2}-\left(1-\delta_{0}\right) C_{3}\left(A_{i j} \frac{\partial v}{\partial x_{j}}, \frac{\partial v}{\partial x_{i}}\right)-\frac{\delta_{0}}{2}|\Phi|^{2},
\end{aligned}
$$

where

$$
C_{3}=\frac{a_{0}}{2\left(9 C_{1}^{2}(A)+1-\delta_{0}\right)} .
$$

From this and the identity (2.26) we obtain

$$
\begin{aligned}
& \left.\sum_{m=0}^{n} \int_{\Omega_{m}}\left\{\rho\left(\frac{\partial u}{\partial t}, \frac{\partial v}{\partial t}\right)+\left(A_{i j} \frac{\partial u}{\partial x_{j}}, \frac{\partial v}{\partial x_{i}}\right)+(B E, \Phi)+\mu(H, \Psi)\right\} d x\right|_{t=0} ^{t=T} \\
& \leq \frac{1}{2} \int_{0}^{T} \int_{S}\left\{\left|\frac{\partial u}{\partial t}+\left(A_{i j} \frac{\partial v}{\partial x_{j}}-A_{i}^{*} \Phi\right) v_{i}\right|^{2}+|\Phi-[v, H]|^{2}\right\} d S d t \\
& -\frac{1}{2} \int_{0}^{T} \int_{S}\left\{\left|\frac{\partial u}{\partial t}\right|^{2}+|[v, H]|^{2}+2\left(1-\delta_{0}\right) C_{3}\left(A_{i j} \frac{\partial v}{\partial x_{j}}, \frac{\partial v}{\partial x_{i}}\right)+\delta_{0}|\Phi|^{2}\right\} d S d t .
\end{aligned}
$$


We multiply (2.24) and (2.25) by

$$
d_{1}=\frac{1}{2 C(\Omega) \max \left\{\rho, \mu^{(n)}\right\}}, \quad d_{2}=\frac{1}{2 C(\Omega) \max \left\{\frac{b_{1}}{\delta_{0}} \frac{1}{2\left(1-\delta_{0}\right) C_{3}}\right\}},
$$

respectively, and add the inequalities thus obtained to (2.30); using the inequality

$$
\begin{aligned}
& \left.\sum_{m=0}^{n} \int_{\Omega_{m}}\left\{\rho\left(\frac{\partial u}{\partial t}, \frac{\partial v}{\partial t}\right)+\left(A_{i j} \frac{\partial u}{\partial x_{j}}, \frac{\partial v}{\partial x_{i}}\right)+(B E, \Phi)+\mu(H, \Psi)\right\} d x\right|_{t=0} ^{t=T} \\
& \quad \leq \mathcal{D}_{0} \sum_{m=0}^{n} \int_{\Omega_{m}}\left\{\rho\left|\frac{\partial u}{\partial t}\right|^{2}+\left(A_{i j} \frac{\partial u}{\partial x_{j}}, \frac{\partial u}{\partial x_{i}}\right)+(B E, E)+\mu|H|^{2}\right. \\
& \left.\quad+\rho\left|\frac{\partial v}{\partial t}\right|^{2}+\left(A_{i j} \frac{\partial v}{\partial x_{j}}, \frac{\partial v}{\partial x_{i}}\right)+(B \Phi, \Phi)+\mu|\Psi|^{2}\right\} d x
\end{aligned}
$$

where

$$
\mathcal{D}_{0}=\max \left\{1, b_{1} b_{0}^{-1}, 9 C(A) C_{0}^{-1}\right\}
$$

we arrive at the estimate

$$
\begin{aligned}
& {\left[d_{1}\left(1-\delta_{1} C_{1}\right)\left(T-T_{1}\right)-\mathcal{D}_{0}\right]\left\|\left\{u, \frac{\partial u}{\partial t}, E, H\right\}\right\|_{\mathcal{H}}^{2}} \\
& +\left[d_{2}\left(1-\delta_{1} C_{1}\right)\left(T-T_{1}\right)-\mathcal{D}_{0}\right]||\left\{v, \frac{\partial v}{\partial t}, \Phi, \Psi\right\} \|_{\widetilde{\mathcal{H}}}^{2} \\
& \leq \frac{1}{2} \int_{0}^{T} \int_{S}\left\{\left|\frac{\partial u}{\partial t}+\left(A_{i j} \frac{\partial v}{\partial x_{j}}-A_{i}^{*} \Phi\right) v_{i}\right|^{2}+|\Phi-[H, v]|^{2}\right\} d S d t .
\end{aligned}
$$

From (2.31) we deduce the following uniqueness property.

Theorem 2.3. Assume that $S_{m}$ and $\Omega$ satisfy conditions (2.17). Suppose that the matrices $A_{i j}^{(m)}, B^{(m)}$ and the coefficients $\mu^{(m)}$ satisfy the monotonicity conditions (2.22), $A_{i j}^{(n)}$ satisfy the condition (2.29),

$$
0<\beta(x) \leq \frac{C_{0} \chi}{C(\Omega)}
$$


Let $f(x) \in M_{1} \cap \mathcal{D}(\mathcal{A}), \varphi(x) \in \tilde{M}_{1} \cap \mathcal{D}(\widetilde{\mathcal{A}})$. Suppose that $\{u(x, t)$, $E(x, t), H(x, t)\}$ and $\{v(x, t), \Phi(x, t), \Psi(x, t)\}$ are solutions of problems (1.2)(1.5) and (1.6)-(1.9) with zero boundary conditions, respectively, and that

$$
\frac{\partial u}{\partial t}+\left(A_{i j} \frac{\partial v}{\partial x_{j}}-A_{i}^{*} \Phi\right) v_{i}=0, \quad \Phi-[H, v]=0 \quad \text { on } \quad S \times(0, T) .
$$

In this case, if $T>T^{*}=T_{1}+\frac{\mathcal{D}_{0}}{1-\delta_{1} C_{1}} \max \left\{\frac{1}{d_{1}}, \frac{1}{d_{2}}\right\}$, then

$$
\begin{gathered}
u(x, t) \equiv E(x, t) \equiv H(x, t) \equiv v(x, t) \equiv \Phi(x, t) \equiv \Psi(x, t) \equiv 0 \\
\text { in } \quad \Omega \times(0, T) .
\end{gathered}
$$

From Theorem 2.3 it follows that for $T>T^{*}$ the expression

$$
\begin{aligned}
\|\{f, \varphi\}\|_{\mathcal{F}} \equiv & \left(\int _ { 0 } ^ { T } \int _ { S } \left\{\left|\frac{\partial u}{\partial t}+\left(A_{i j} \frac{\partial v}{\partial x_{j}}-A_{i}^{*} \Phi\right) v_{i}\right|^{2}\right.\right. \\
+ & \left.\left.|\Phi-[H, v]|^{2}\right\} d S d t\right)^{\frac{1}{2}}
\end{aligned}
$$

defines a norm on the set of initial data $f=\left\{f_{1}, f_{2}, f_{3}, f_{4}\right\}$ and $\varphi=\left\{\varphi_{1}, \varphi_{2}, \varphi_{3}, \varphi_{4}\right\}$ of problems (1.2)-(1.5) and (1.6)-(1.9) with zero boundary conditions. In (2.32)

$$
\left\{u, \frac{\partial u}{\partial t}, E, H\right\}=\mathcal{U}(t) f, \quad\left\{v, \frac{\partial v}{\partial t}, \Phi, \Psi\right\}=\widetilde{U}(t) \varphi .
$$

We denote by $\mathcal{F}$ the Hilbert space obtained by completing $M_{1} \cap \mathcal{D}(\mathcal{A}) \times \widetilde{M}_{1} \cap$ $\mathcal{D}(\widetilde{\mathcal{A}})$ with respect to the norm (2.32). We have

$$
\|\{f, \varphi\}\|_{\mathcal{E}}^{2} \equiv\|f\|_{\mathcal{H}}^{2}+\|\varphi\|_{\widetilde{\mathcal{H}}}^{2} \leq C\|\{f, \varphi\}\|_{\mathcal{F}}^{2}, \quad \mathcal{F} \subset \mathcal{E} \equiv \mathcal{H} \times \widetilde{\mathcal{H}} .
$$

Our next purpose is to prove simultaneous exact controllability for the problems (1.2)-(1.5), (1.6)-(1.9). 


\section{Exact controllability}

We denote by $\mathcal{F}^{\prime}$ the dual space of $\mathcal{F}$ with respect to $\mathcal{E}$. Let us consider the pair of problems: (1.2)-(1.4) with boundary conditions

$$
\begin{array}{r}
\left.\left\{\left(A_{i j} \frac{\partial u}{\partial x_{j}}-A_{i}^{*} E\right) v_{1}+\beta u\right\}\right|_{S \times(0, T)} \\
=Q(x, t),\left.[E, \nu]\right|_{S \times(0, T)}=[v, \mathcal{P}]
\end{array}
$$

and (1.6)-(1.8) with boundary conditions

$$
\left.\frac{\partial v}{\partial t}\right|_{S \times(0, T)}=Q(x, t),\left.\quad[\Psi, v]\right|_{S \times(0, T)}=\mathcal{P}(x, t),
$$

where $Q(x, t), \mathcal{P}(x, t) \in L_{2}(S \times(0, T)),\{f(x), \varphi(x)\} \in \mathcal{F}^{\prime}$.

We rewrite systems (1.2) and (1.6) in the form

$$
\frac{d}{d t}\left\{u, u^{\prime}, E, H\right\}=\mathcal{A}\left\{u, u^{\prime}, E, H\right\}, \quad \frac{d}{d t}\left\{v, v^{\prime}, \Phi, \Psi\right\}=\widetilde{\mathcal{A}}\left\{v, v^{\prime}, \Phi, \Psi\right\}
$$

By definition,

$$
\left\{u(t), u^{\prime}(t), E(t), H(t), v(t), v^{\prime}(t), \Phi(t), \Psi(t)\right\} \in L_{\infty}\left(0, T ; \mathcal{F}^{\prime}\right)
$$

is a solution of (1.2)-(1.4), (3.1) and (1.6)-(1.8), (3.2) if the identity

$$
\begin{aligned}
& \left\langle\left\{u(t), u^{\prime}(t), E(t), H(t), v(t), v^{\prime}(t), \Phi(t), \Psi(t)\right\},\{\mathcal{U}(t) \tilde{f}, \widetilde{U}(t) \tilde{\varphi}\}\right\rangle_{\mathcal{E}} \\
& =\int_{0}^{t} \int_{S}\left\{\left(Q, \frac{\partial \widetilde{u}}{\partial \tau}+\left(A_{i j} \frac{\partial \widetilde{v}}{\partial x_{j}}-A_{i}^{*} \widetilde{\Phi}\right) v_{i}\right)\right. \\
& \quad+(\mathcal{P},[\tilde{H}, v]-\widetilde{\Phi})\} d S d \tau+\langle\{f, \varphi\},\{\tilde{f}, \widetilde{\varphi}\}\rangle_{\mathcal{E}}
\end{aligned}
$$

holds for all $\{\tilde{f}, \tilde{\varphi}\} \in \mathcal{F}, 0<t<T$. In (3.3)

$$
\begin{aligned}
& \langle\{f, \varphi\},\{\tilde{f}, \widetilde{\varphi}\}\rangle_{\mathcal{E}}=\langle f, \widetilde{f}\rangle_{\mathcal{H}}+\langle\varphi, \widetilde{\varphi}\rangle_{\widetilde{\mathcal{H}}}, \\
& \left\{\widetilde{u}, \frac{\partial \widetilde{u}}{\partial t}, \widetilde{E}, \widetilde{H}\right\}=\mathcal{U}(t) \widetilde{f}, \quad\left\{\widetilde{v}, \frac{\partial \widetilde{v}}{\partial t}, \widetilde{\Phi}, \widetilde{\Psi}\right\}=\widetilde{U}(t) \widetilde{\varphi} .
\end{aligned}
$$

In a similar way we define a solution of (1.2), (1.4), (3.1) and (1.6), (1.8), (3.2) with zero data for $t=T$ :

$$
\left\{u(t), u^{\prime}(t), E(t), H(t), v(t), v^{\prime}(t), \Phi(t), \Psi(t)\right\} \in L_{\infty}\left(0, T ; \mathcal{F}^{\prime}\right)
$$


is a solution of (1.2), (1.4), (3.1), (1.6), (1.8), (3.2) with zero data for $t=T$ if

$$
\begin{aligned}
& \left\langle\left\{u(t), u^{\prime}(t), E(t), H(t), v(t), v^{\prime}(t), \Phi(t), \Psi(t)\right\},\{\mathcal{U}(t) \tilde{f}, \widetilde{U}(t) \widetilde{\varphi}\}\right\rangle_{\mathcal{E}} \\
& =-\int_{t}^{T} \int_{S}\left\{\left(Q, \frac{\partial \widetilde{u}}{\partial t}+\left(A_{i j} \frac{\partial \widetilde{v}}{\partial x_{j}}-A_{i}^{*} \widetilde{\Phi}\right) v_{i}\right)\right. \\
& \quad+(\mathcal{P},[\widetilde{H}, v]-\widetilde{\Phi})\} d S d \tau
\end{aligned}
$$

for all $\{\tilde{f}, \tilde{\varphi}\} \in \mathcal{F}, 0<t<T$.

Let $\{g, \psi\}$ be an arbitrary element of $\mathcal{F}$, and let $\left\{u, u^{\prime}, E, H, v, v^{\prime}, \Phi, \Psi\right\}$ be solution of (1.2), (1.4), (3.1), (1.6), (1.8), (3.2) with zero data for $t=T, T>T^{*}$, and boundary functions

$$
Q=-\left(\frac{\partial w}{\partial t}+\left(A_{i j} \frac{\partial m}{\partial x_{j}}-A_{i}^{*} p\right) v_{i}\right), \mathcal{P}=-([h, v]-p),
$$

where

$$
\left\{w, \frac{\partial w}{\partial t}, e, h\right\}=\mathcal{U}(t) g, \quad\left\{m, \frac{\partial m}{\partial t}, p, q\right\}=\tilde{U}(t) \psi .
$$

We set

$$
M\{g, \psi\}=\left.\left\{u, u^{\prime}, E, H, v, v^{\prime}, \Phi, \Psi\right\}\right|_{t=0} .
$$

From (3.4) it follows that

$$
\langle M\{g, \psi\},\{\tilde{f}, \tilde{\varphi}\}\rangle_{\mathcal{E}}=\langle\{g, \psi\},\{\tilde{f}, \tilde{\varphi}\}\rangle_{\mathcal{F}}
$$

for any $\{\tilde{f}, \tilde{\varphi}\} \in \mathcal{F}$. This implies that $M$ is an isomorphism of $\mathcal{F}$ onto the whole of $\mathcal{F}^{\prime}$.

We return to problems (1.2)-(1.4), (3.1) and (1.6)-(1.8), (3.2). Suppose that the initial data $\{f, \varphi\}$ belong to $\mathcal{F}^{\prime}$. We set

$$
\begin{gathered}
\{g, \psi\}=M^{-1}\{f, \varphi\}, \\
Q=-\left(\frac{\partial w}{\partial t}+\left(A_{i j} \frac{\partial m}{\partial x_{j}}-A_{i}^{*} p\right) v_{i}\right), \quad \mathcal{P}=-([h, \nu]-p),
\end{gathered}
$$

where

$$
\left\{w, \frac{\partial w}{\partial t}, e, h\right\}=\mathcal{U}(t) g, \quad\left\{m, \frac{\partial m}{\partial t}, p, q\right\}=\widetilde{U}(t) \psi .
$$


From (3.3) with $t=T>T^{*}$ we find that

$$
\begin{aligned}
& \left\langle\left\{u(T), u^{\prime}(T), E(T), H(T), v(T), v^{\prime}(T), \Phi(T), \Psi(T)\right\},\{\mathcal{U}(T) \tilde{f}, \widetilde{U}(T) \widetilde{\varphi}\}\right\rangle_{\mathcal{E}} \\
& \quad=\langle M\{g, \psi\},\{\tilde{f}, \widetilde{\varphi}\}\rangle_{\mathcal{E}}-\langle\{g, \psi\},\{\tilde{f}, \widetilde{\varphi}\}\rangle_{\mathcal{F}}
\end{aligned}
$$

for any $\{\tilde{f}, \widetilde{\varphi}\} \in \mathcal{F}$. By (3.5), the right-hand side of the last identity is equal to zero; that is, $\left\{u(T), u^{\prime}(T), E(T), H(T), v(T), v^{\prime}(T), \Phi(T), \Psi(T)\right\}$ generates the zero functional on $\mathcal{F}$.

We arrive at the following assertion.

Theorem 3.1. Assume that $A_{i j}^{(m)}, B^{(m)}, \mu^{(m)}, S_{m}$ and $\Omega$ satisfy the conditions of Theorem 2.3. If $T>T^{*}$, then for any initial data $\{f, \varphi\} \in \mathcal{F}^{\prime}$ of problems (1.2)-(1.5) and (1.6)-(1.9) there exists a control $\{\mathcal{D}(x, t), \mathcal{P}(x, t)\} \in$ $C^{1}\left(0, T ; L_{2}(S)\right) \times C^{0}\left(0, T ; L_{2}(S)\right)$ such that the corresponding solution of problem (1.6)-(1.9) satisfies

$$
\left.\left\{v, \frac{\partial v}{\partial t}, \Phi, \Psi\right\}\right|_{t=T}=0,
$$

while the vector-valued functions

$$
Q(x, t)=\frac{\partial}{\partial t} \mathcal{D}(x, t), \quad G(x, t)=[v, \mathcal{P}(x, t)]
$$

drive the system (1.2)-(1.5) to a state of rest at the same time $T$ :

$$
\left.\left\{u, \frac{\partial u}{\partial t}, E, H\right\}\right|_{t=T}=0
$$

To prove this assertion, it suffices to construct functions $Q(x, t), \mathcal{P}(x, t)$ as before, by setting

$$
\mathcal{D}(x, t)=\int_{0}^{t} Q(x, \tau) d \tau+\varphi_{1}(x) .
$$

We remark that, in view of the linearity of the systems, it suffices to consider controls that reduce the systems to a state of rest. 


\section{REFERENCES}

[1] F. Alabau and V. Komornik, Boundary observability, controllability and stabilization of linear elastodynamic systems, Institut de Recherche Mathématique Avancée, Preprint, Series 15, (1996).

[2] A. Borrelli and M.C. Patria, Spatial energy estimates in dynamical problems for a semi-infinite piezoelectric beam, Journal of Applied Mathematics, 64 (2000), pp. 73-93.

[3] G. Duvaut and J.-L. Lions, Les Inéquations en M'ecanique et en Physique, Dunod, Paris, (1972).

[4] J.N. Eringen and G.A. Maugin, Electrodynamics of continua, Vol. 1,2 (1990), Berlin, Springer.

[5] M.A. Horn, Implications of sharp trace regularity results on boundary stabilization of the system of linear elasticity, J. Math. Anal. Appl., 223 (1998), pp. 126-150.

[6] T. Ikeda, Fundamentals of Piezoelectricity, Oxford, University Press, (1996).

[7] B.V. Kapitonov, Uniqueness theorem and exact boundary controllability of evolution systems, Siberian Math. J., 34 (1993), pp. 852-868.

[8] B.V. Kapitonov, Stabilization and exact boundary controllability for Maxwell's equations, SIAM J. Control Optim., 32 (1994), pp. 408-421.

[9] B.V. Kapitonov, Uniform stabilization and simultaneous exact boundary controllability for a pair of hyperbolic systems, Siberian Math. J., 35 (4) (1994), pp. 722-734.

[10] B.V. Kapitonov, Simultaneous precise control of systems of linear elasticity, Russian Acad. Sci. Dokl. Math., 48 (2) (1994), pp. 332-337.

[11] B.V. Kapitonov, Stabilization and simultaneous boundary controllability for a pair of Maxwell's equations, Comp. Appl. Math., 15 (3) (1996), pp. 213-225.

[12] B.V. Kapitonov, Stabilization and simultaneous boundary controllability for a class of evolution systems, Comp. Appl. Math., 17 (1998), pp. 149-160.

[13] B.V. Kapitonov, Simultaneous exact controllability for a class of evolution systems, Comp. Appl. Math., 18 (2) (1999), pp. 149-161.

[14] B.V. Kapitonov and M.A. Raupp, Exact boundary controllability in problems of transmission for the system of electromagneto-elasticity, Math. Meth. Appl. Sci., 24 (2001), pp. 193-207.

[15] F.A. Khodja and A. Bader, Stabilizability of systems of one-dimensional wave equations by one internal or boundary control force, SIAM J. Control Optim., 39 (6) (2001), pp. 18331851.

[16] K.A. Kime, Boundary controllability of Maxwell's equations in a spherical region, SIAM J. Control Optim., 28 (1990), pp. 294-319.

[17] V. Komornik, Boundary stabilization, observation and control of Maxwell's equations, Panamer. Math. J., 4 (1994), pp. 47-61. 
[18] J.E. Lagnese, Boundary stabilization of linear elastodynamic systems, SIAM J. Control Optim., 21 (6) (1983), pp. 968-984.

[19] J.E. Lagnese, Boundary controllability in problems of transmission for a class of second order hyperbolic systems, ESAIM: Control, Optim. and Calculus of Variations, 2 (1997), pp. 343-357.

[20] J.E. Lagnese, Exact boundary controllability of Maxwell's equations in a general region, SIAM J. Control Optim., 27 (1989), pp. 374-388.

[21] J.-L. Lions, Contrôlabilité exacte des systémes distribués, C.R. Acad. Sci. Paris, Ser. I Math. 302 (1986), pp. 471-475.

[22] J.-L. Lions, Exact controllability, stabilization and perturbations for distributed systems, SIAM Rev., 30 (1988), pp. 1-68.

[23] J.-L. Lions, Contrôlabilité Exacte, Perturbations et Stabilisation des Systèmes Distribués, Tome 1. Contrôlabilite Exacte, Coll. RMA, V. 8 (1998), Masson, Paris.

[24] P. Martinez, Boundary stabilization of the wave equation in almost star-shaped domains, SIAM J. Control Optim., 37 (3) (1999), pp. 673-694.

[25] S. Nicaise, Boundary exact controllability of interface problems with singularities. I: addition of the coefficients of singularities, SIAM J. Control Optim., 34 (1996), pp. 1512-1532.

[26] S. Nicaise, Boundary exact controllability of interface problems with singularities. II: addition of internal control, SIAM J. Control Optim., 35 (1997), pp. 585-603.

[27] D.L. Russell, Dirichlet-Neumann boundary control problem associated with Maxwell's equations in a cylindrical region, SIAM J. Math. Anal., 24 (1986), pp. 199-229.

[28] N. Weck, Exact boundary controllability of a Maxwell problem, SIAM J. Control Optim., 38 (2000), pp. 736-750. 